\title{
Testing for Parameter Constancy Using Chebyshev Time Polynomials
}

\author{
Luis F. Martins* \\ June 7, 2009
}

\begin{abstract}
We propose a simple method of testing for parameter constancy in regression models that allow for coefficients that vary smoothly over time. The model is related to Bierens and Martins (2009) but in our case we consider stationary processes. The procedure is shown to have good statistical properties. We revisited Hansen's (2001) study of structural breaks in a AR(1) model of labor productivity in the U.S. manufacturing/durables sector and found evidence of time-varying autoregressive parameter.

Keywords: Parameter Constancy, Chebyshev Time Polynomials, Linear Regression.

J.E.L. Classification: C22.
\end{abstract}

*UNIDE and Department of Quantitative Methods, ISCTE - Business School, Av. das Forças Armadas, 1649-026 Lisbon, Portugal. E-mail: luis.martins@iscte.pt. Financial support under grant PTDC/ECO/68367/2006 from the Fundação para a Ciência e Tecnologia is gratefully acknowledged. 


\section{Introduction}

Recently, Clive W. J. Granger, making use of a theorem due to Halbert White, claimed that "any non-linear model can be approximated by a timevarying parameter linear model". In his argument, the time-varying parameter (hereafter TV) "could be a deterministic function of time", among other stochastic alternatives (see Granger, 2008, for details). This principle was implicitly introduced by Bierens and Martins (2009) (henceforth BM) and Park and Hahn (1999) in TV cointegrated relationships. In BM, the TV cointegrating vector was approximated by a linear combination of orthogonal Chebyshev time polynomials so that the resulting vector error correction model had time invariant coefficients. Park and Hahn's (1999) cointegrating model is in levels and the approximation is by means of arbitrary orthogonal Fourier functions. In this paper, we follow Halbert White and Granger's (2008) principle and model a stable autoregressive process with a TV autoregressive coefficient approximated by a combination of Chebyshev polynomials, as in BM. It is shown analytically that, for large samples, least squares does not suffer from the collinearity nor the endogeneity issues.

Failure to detect and account for parameter shifts in a model is a serious form of misspecification. Therefore, several testing procedures for parameter constancy have been proposed in the literature ${ }^{1}$. When there is a sudden single break at an unknown period of time, the most popular statistics are the "Sup", suggested by Quandt (1960) and Andrews (1993), and the "ExpWald" with stronger optimality properties of Andrews and Ploberger (1994). When it is allowed for more than one sudden unknown break, the "UD max" and "WD max" statistics of Bai and Perron (1998) is a preferable alternative. On the other hand, following the work developed by Nyblom (1989), who considered time-variation of the form of a martingale process, Hansen (1992) proposed the " $L_{c}$ " statistic based on partial sums of the least squares normal equations. In this study, we propose a standard $F$-test for parameter constancy that follows straightforwardly from the suggested regression with Chebyshev polynomials. Contrary to the two above-mentioned strands of literature, the rejection of the parameter constancy hypothesis in our testing procedure might indicate evidence for a parameter that varies smoothly and deterministically over time. According to a limited Monte Carlo study, the test performs well in finite samples.

\footnotetext{
${ }^{1}$ For comprehensive reviews see Hansen (2001) and Perron (2006).
} 
We apply our methodology to the U.S. labor productivity data. For those series for which Hansen (2001) found evidence of structural breaks in a $\mathrm{AR}(1)$ model using the standard tests, we conclude in a similar fashion but with respect to deterministic TV coefficients. Hence, our AR(1) model with TV autoregressive coefficient might be a reasonable specification for this data, giving credit to Clive W. J. Granger's conjecture. The remainder of the paper is organized as follows. In Section 2 we introduce the TV AR(1) model and the test statistic. In Section 3 we study the empirical size and power properties of the test via Monte Carlo simulations. In Section 4, we illustrate the merits of our approach by applying the procedures to the U.S. labor productivity data and we conclude.

\section{Testing for Parameter Constancy}

In the standard linear regression model $y_{t}=x_{t}^{\prime} \beta+u_{t}$, where the observed time-series $\left(y_{t}, x_{t}^{\prime}\right)_{t=1}^{T}$ is assumed to be stationary, the $k \times 1$ parameter vector $\beta$ is time invariant and $u_{t}$ satisfies some functional central limit theorem. We relax this time-invariance property of $\beta$ by modeling it as a TV vector $\beta_{t}$ according to a deterministic law using Chebyshev time polynomials $P_{i, T}(t)$, defined by

$$
\begin{aligned}
P_{0, T}(t) & =1, P_{i, T}(t)=\sqrt{2} \cos (i \pi(t-0.5) / T) \\
t & =1,2, \ldots, T, i=1,2,3, \ldots
\end{aligned}
$$

as in BM. The polynomials are orthonormal, in the sense that for all integers $i, j, \frac{1}{T} \sum_{t=1}^{T} P_{i, T}(t) P_{j, T}(t)=1(i=j)$, where $1(\cdot)$ is the indicator function. Due to this orthonormality property, any function of time $g(t), t=1, \ldots, T$ can be represented by

$$
g(t)=\sum_{i=0}^{T-1} \xi_{i, T} P_{i, T}(t), \text { where } \xi_{i, T}=\frac{1}{T} \sum_{t=1}^{T} g(t) P_{i, T}(t) .
$$

Here, $g(t)$ is linearly decomposed in components $\xi_{i, T} P_{i, T}(t)$ of decreasing smoothness and may be approximated quite well by

$$
g_{m}(t)=\sum_{i=0}^{m} \xi_{i, T} P_{i, T}(t)
$$


for some relatively small fixed natural number $m \ll T-1 \quad 2$.

\subsection{Time Varying AR(1) Model}

For sake of exposition, we discuss the case of time-variation in the slope of the first-order autoregression:

$$
y_{t}=\alpha+\rho_{t} y_{t-1}+u_{t}, t=1, \ldots, T,
$$

where $u_{t} \sim$ i.i.d. $\left(0, \sigma_{u}^{2}\right)$ and $\left|\rho_{t}\right|<1$, for all $t$, so that $y_{t}$ is bounded in probability. Granger (2008) calls this process "stable (or stationary)" and distinguishes the strong from the weak form of stability ${ }^{3}$. Doing backwards substitution, we get

$$
y_{t}=\alpha\left(1+\sum_{l=0}^{t} \prod_{k=0}^{l} \rho_{t-k}\right)+y_{0} \prod_{k=0}^{t-1} \rho_{t-k}+u_{t}+\sum_{l=1}^{t} u_{t-l} \prod_{k=1}^{l} \rho_{t-k+1},
$$

which implies TV unconditional moments with $\sigma_{t}^{2}=\rho_{t}^{2} \sigma_{t-1}^{2}+\sigma_{u}^{2}$, where

$$
V\left(y_{t}\right)=\sigma_{u}^{2}\left(1+\sum_{l=0}^{t-1} \prod_{k=0}^{l} \rho_{t-k}^{2}\right) \equiv \sigma_{t}^{2} .
$$

With $P_{i, T}(t)$ spanning an Hilbert space, we may denote $\rho_{t}$ as $\rho_{t}=$ $\sum_{i=0}^{T-1} \xi_{i, T} P_{i, T}(t)$, where $\xi_{i, T}=\frac{1}{T} \sum_{t=1}^{T} \rho_{t} P_{i, T}(t), i=0, \ldots, T-1$, are unknown scalars. The null hypothesis of parameter constancy is, therefore, $\xi_{i, T}=0$ for $i=1, \ldots, T-1$; whereas the TV alternative of structural breaks we consider is such that $\lim _{T \rightarrow \infty} \xi_{i, T} \neq 0$ for some $i=1, \ldots, m$, where $m \ll T-1$ is chosen in advance. Substituting $\rho_{t}=\sum_{i=0}^{m} \xi_{i, T} P_{i, T}(t)$ in (4) yields

$$
y_{t}=\alpha+\xi^{\prime} y_{t-1}^{(m)}+v_{t}, t=1, \ldots, T
$$

where $\xi^{\prime}=\left(\xi_{0}, \xi_{1}, \ldots, \xi_{m}\right) \in \Re^{m+1}$ are now time-invariant coefficients and $y_{t-1}^{(m)}$ is defined by $y_{t-1}^{(m)}=P_{T}(t) y_{t-1}$, with $P_{T}^{\prime}(t)=\left(1, P_{1, T}(t), \ldots, P_{m, T}(t)\right)$. The following Theorem provides a sufficient condition for the roots of $\left(1-\xi^{\prime} P_{T}(t) L\right)$ lie outside the unit circle for all $t$.

\footnotetext{
${ }^{2}$ See Bierens and Martins (2009), Section 2.2, for technical details.

${ }^{3}$ In the strong form, $\left|\rho_{t}\right|<1$, for all $t$, whereas in the weak version, $\left|\rho_{t}\right|<1$, for all $t$ such that $\left|Y_{t}\right|>k$, where $k$ is some positive number.
} 
Lemma 1. In model (7), if $\sum_{i=0}^{m} \xi_{i, T}^{2}<\frac{1}{2(m+1)}$ then $\left|\sum_{i=0}^{m} \xi_{i, T} P_{i, T}(t)\right|<1$, for all $t$ and a fixed $m$ and $T$.

Proof: Apply Holders's inequality to $\sum_{i=0}^{m}\left|\xi_{i, T} P_{i, T}(t)\right|$ and note that $\sum_{i=0}^{m} P_{i, T}(t)^{2}$ is smaller than $2(m+1)$, for all $t$, for any fixed $m$ and $T$.

\subsection{Testing Procedure}

The test for parameter constancy is a standard one and results from transforming the original model (4), with a TV coefficient, to model (7), with time-invariant coefficients, given the properties of the Chebyshev time polynomials. Under the null hypothesis of slope constancy in the $\operatorname{AR}(1)$ model, $\xi^{\prime}=\left(\rho, O_{1 \times m}\right)$, so that $\xi^{\prime} y_{t-1}^{(m)}=\rho y_{t-1}$. This suggests estimating model (7) with least squares and do a right-sided joint significance $F$-test to $\xi$. We denote this statistic by $F_{m, T}$, which has a $F$-distribution, under the null, and that correspondents to an asymptotic chi-square test procedure.

\section{$3 \quad$ Size and Power Analysis}

To conduct the empirical size analysis we applied our $F_{m, T}$ test to 10,000 replications of the model $y_{t}=\alpha+\rho y_{t-1}+u_{t}, t=1, \ldots, T$, where $\alpha=0$ and $u_{t}$ is drawn independently from the standard normal distribution, for various values of $\rho, T$ and $m$. The results are given in Table 1 . In each entry, we have the non-acceptance frequencies based on the $F$ critical values for a size of $5 \%$. For a matter of comparison, we also computed the size of the tests $L_{c}$, SupW, ExpW and $U D \max$. The $S u p W$ test under-rejects the null hypothesis and the $U D$ max performs poorly in terms of size.

The $F_{m, T}$ test tends to over-accept the hypothesis of parameter constancy, specially for large values of $m$. The intuition is the following. For $T$ fixed, it can be easily shown that $F_{m, T}=\left(\frac{S S R_{R}}{S S R_{U R}}-1\right)\left(\frac{T-1}{m}-1\right)$, where $^{4}$

$$
\frac{1}{T} S S R_{U R}=\sum_{i=0}^{m} \widehat{\xi}_{i}^{2} \frac{1}{T} \sum_{t=2}^{T} P_{i, T}(t)^{2} y_{t-1}^{2}-2 \sum_{i=0}^{m} \widehat{\xi}_{i} \frac{1}{T} \sum_{t=2}^{T} P_{i, T}(t) y_{t-1} u_{t}+\frac{1}{T} \sum_{t=2}^{T} u_{t}^{2} .
$$

\footnotetext{
${ }^{4} S S R_{R}$ does not depend on $m$ and we assume $\rho=0$ without loss of generality.
} 
Table 1: Empirical Size Analysis

\begin{tabular}{|c|c|c|c|c|c|c|c|c|c|}
\hline$m$ & $\rho \backslash T$ & 50 & 100 & 200 & & 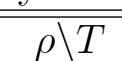 & 50 & 100 & 200 \\
\hline \multirow[t]{4}{*}{1} & -0.25 & 0.037 & 0.044 & 0.047 & $L_{c}$ & -0.25 & 0.043 & 0.046 & 0.044 \\
\hline & 0 & 0.038 & 0.044 & 0.047 & & 0 & 0.042 & 0.047 & 0.047 \\
\hline & 0.25 & 0.037 & 0.045 & 0.049 & & 0.25 & 0.042 & 0.047 & 0.048 \\
\hline & 0.75 & 0.036 & 0.044 & 0.046 & & 0.75 & 0.032 & 0.036 & 0.041 \\
\hline \multirow[t]{4}{*}{$T / 10$} & -0.25 & 0.017 & 0.015 & 0.010 & SupW & -0.25 & 0.014 & 0.020 & 0.029 \\
\hline & 0 & 0.017 & 0.015 & 0.009 & & 0 & 0.014 & 0.020 & 0.029 \\
\hline & 0.25 & 0.0 & 4 & 0.00 & & 0.25 & 0.014 & 0.020 & 0.031 \\
\hline & 0.75 & 0.026 & 0.023 & 0.022 & & 0.75 & 0.020 & 0.028 & 0.037 \\
\hline \multirow[t]{4}{*}{$T / 5$} & -0.25 & 0.008 & 0.003 & 0.001 & $\operatorname{Exp} W$ & -0.25 & 0.027 & 0.033 & 0.040 \\
\hline & 0 & 0.007 & 0.002 & 0.001 & & 0 & 0.026 & 0.033 & 0.040 \\
\hline & 0.25 & 0.009 & 0.004 & 0.001 & & 0.25 & 0.027 & 0.033 & 0.043 \\
\hline & 0.75 & 0.025 & 0.019 & 0.014 & & 0.75 & 0.031 & 0.036 & 0.042 \\
\hline \multirow[t]{4}{*}{$T / 2$} & -0.25 & 0.007 & 0.002 & 0.000 & $U D \max$ & -0.25 & 0.616 & 0.128 & 0.023 \\
\hline & 0 & & 0.0 & 0.000 & & 0 & 0.618 & 0.123 & 0.019 \\
\hline & 0.25 & 0.008 & 0.003 & 0.000 & & 0.25 & 0.620 & 0.125 & 0.022 \\
\hline & 0.75 & 0.012 & 0.009 & 0.004 & & 0.75 & 0.618 & 0.156 & 0.034 \\
\hline
\end{tabular}


Table 2: Empirical Power Analysis

\begin{tabular}{|c|c|c|c|c|c|c|c|c|c|}
\hline$m$ & $\bar{T}$ & 50 & 100 & 200 & & $\bar{T}$ & 50 & 100 & 200 \\
\hline \multirow[t]{2}{*}{1} & $M 1$ & 0.993 & 1.000 & 1.000 & $L_{c}$ & $M 1$ & 0.987 & 1.000 & 1.000 \\
\hline & $M 2$ & 0.780 & 0.994 & 1.000 & & $M 2$ & 0.071 & 0.003 & 0.000 \\
\hline \multirow[t]{2}{*}{$T / 10$} & $M 1$ & 0.964 & 0.999 & 1.000 & SupW & $M 1$ & 0.972 & 1.000 & 1.000 \\
\hline & $M 2$ & 1.000 & 1.000 & 1.000 & & $M 2$ & 1.000 & 1.000 & 1.000 \\
\hline \multirow[t]{2}{*}{$T / 5$} & $M 1$ & 0.902 & 0.997 & 1.000 & $\operatorname{Exp} W$ & $M 1$ & 0.989 & 1.000 & 1.000 \\
\hline & $M 2$ & 1.000 & 1.000 & 1.000 & & $M 2$ & 1.000 & 1.000 & 1.000 \\
\hline \multirow[t]{2}{*}{$T / 2$} & $M 1$ & 0.639 & 0.938 & 0.998 & $U D \max$ & $M 1$ & 0.972 & 0.999 & 1.000 \\
\hline & $M 2$ & 1.000 & 1.000 & 1.000 & & $M 2$ & 1.000 & 1.000 & 1.000 \\
\hline
\end{tabular}

This quantity will increase with $m$ because the second term is approximately zero as it is a weighted sample covariance of $y_{t-1}$ and $u_{t}$. Hence, for $T$ fixed, the simulation results suggest that for larger $m$ the statistic $F_{m, T}$ decreases at a faster rate than the $5 \%$ critical value from the $F_{m, T-m-1}$ distribution. For practitioners, the results suggest applying the $F_{m, T}$ test with a relatively small $m$ (This phenomenon is also described at BM.)

Next, we check via a limited Monte Carlo study how the tests perform under TV AR(1) specifications. In Model $1(\mathrm{M} 1), \rho(t / T)$ is a S-shaped function on $[0,1], \rho(x)=6 x^{2}-4 x^{3}-1$. In M2, $\rho(t / T)$ is a combination of Fourier functions, $\rho(x)=x+x^{2}+\cos (2 \pi x)+\sin (2 \pi x)+\cos (4 \pi x)+\sin (4 \pi x)$ in which $\rho(x)$ is non-monotonic and is not inside the $[0,1]$ interval for $x$ close to zero and one. The results are in Table 2 and, in general, the tests perform very well. The exception is $L_{c}$ in Model 2.

\section{Application and Discussion}

In his 2001 paper, Bruce Hansen found strong evidence of structural breaks in a $\mathrm{AR}(1)$ model of labor productivity in the U.S. manufacturing/durables sector. Using monthly data from February 1947 through April 2001 and the standard parameter constancy tests named above, he found support for structural change in the stationary $\mathrm{AR}(1)$ regression for seven of the ten two-digit SIC industries of the manufacturing/durables group: Industry machinery (SIC 35) and Electronic equipment (SIC 36) at the mean growth rate, $\frac{\alpha}{1-\rho}$; Furniture (SIC 25), Primary metals (SIC 33), Fabricated metals (SIC 34), Instruments (SIC 38) and Miscellaneous (SIC 39) at $\rho$. Parameter 
Table 3: $F_{m, T}$ Tests for Parameter Constancy: $p$-values

\begin{tabular}{cccccc}
\hline \hline & $m=1$ & $m=2$ & $m=3$ & $m=4$ & $m=5$ \\
\hline SIC 24 & 0.938 & 0.731 & 0.488 & 0.628 & 0.396 \\
SIC 25 & 0.002 & 0.000 & 0.002 & 0.000 & 0.000 \\
SIC 32 & 0.259 & 0.465 & 0.199 & 0.229 & 0.344 \\
SIC 33 & 0.190 & 0.004 & 0.001 & 0.000 & 0.000 \\
SIC 34 & 0.027 & 0.087 & 0.165 & 0.086 & 0.148 \\
SIC 35 & 0.071 & 0.002 & 0.000 & 0.000 & 0.000 \\
SIC 36 & 0.000 & 0.000 & 0.000 & 0.000 & 0.000 \\
SIC 37 & 0.088 & 0.226 & 0.239 & 0.377 & 0.519 \\
SIC 38 & 0.000 & 0.000 & 0.000 & 0.000 & 0.000 \\
SIC 39 & 0.000 & 0.000 & 0.000 & 0.000 & 0.000 \\
\hline \hline
\end{tabular}

constancy of found for Lumber (SIC 24), Stone, clay and glass products (SIC 32) and Transportation equipment (SIC 37) (see Hansen, 2001, for details). For the same dataset, we extend Hansen's study by modeling the variables according to our TV AR(1) model (7) and testing for parameter constancy by means of our $F_{m, T}$ statistic. The p-values of the tests are presented in Table 3. Small values for $m$ were chosen due to the empirical size and power properties of the test, according to the Monte Carlo experiments in the previous section.

Our results confirm those by Hansen (2001). Whenever the standard tests find evidence for parameter constancy so does our $F_{m, T}$ test. The cases of structural changes are also identified by our test procedure. We also computed the time-sequence $\widehat{\rho}_{t}(m)=\sum_{i=0}^{m} \widehat{\xi}_{i, T} P_{i, T}(t), t=1, \ldots, T$ as a way to model series with TV autoregressive parameter according to (7). For the series for which Bruce Hansen found evidence for breaks at the mean growth rate, we observed that $\widehat{\rho}_{t}(m)$ tended to increase slightly with time. For the cases of changes at $\rho$, the sequence $\widehat{\rho}_{t}(m)$ tended to decrease at a faster rate. To illustrate, we present the sequence $\widehat{\rho}_{t}(m)$ for SIC 38 for $m=1,3$ and 5 at Figure 1. In this case, the curve $\widehat{\rho}_{t}(m)$ is very similar for different $m$ and it drops from 0.15 at the beginning of the sample to -0.4 at the end of the sample $^{5}$. With this empirical application we show that our model (7) can be

\footnotetext{
${ }^{5}$ The sufficient condition $\sum_{i=0}^{m} \xi_{i, T}^{2}<\frac{1}{2(m+1)}$ is satisfied, when $\xi_{i, T}^{2}$ is replaced by its estimate $\widehat{\xi}_{i, T}^{2}$.
} 
suitable for some time-series and that the $F_{m, T}$ test is a valid alternative to the standard tests for parameter constancy.

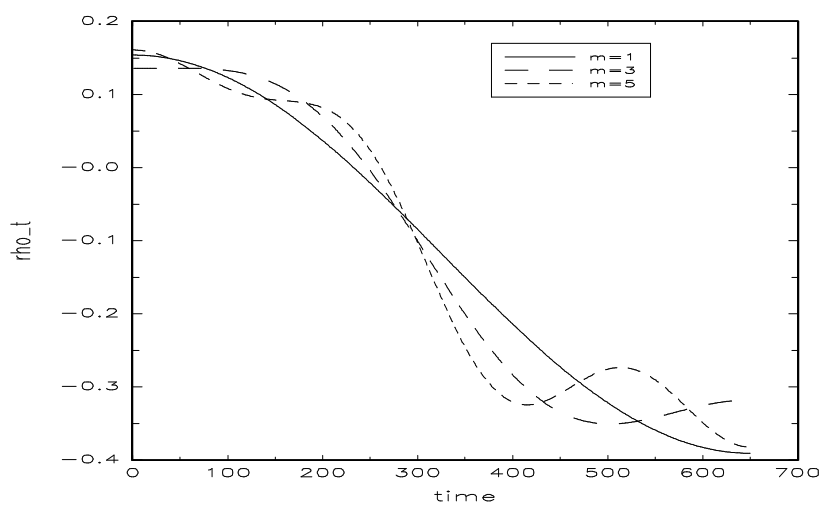

Figure 1. Sequence $\widehat{\rho}_{t}(m)$ for SIC 38 .

Finally, we discuss the issues of (possible) colinearity and endogeneity in model (7). The following Theorem makes proof that there is no colinearity problem in the testing regression (7) for large samples.

Lemma 2. Let

$$
a_{i j}=\int_{0}^{1}[(i \pi) \sin (i \pi x) \cos (j \pi x)+(j \pi) \sin (j \pi x) \cos (i \pi x)] x d x,
$$

for $i, j=0,1,2, \ldots$ Then,

$$
a_{i j}=\left\{\begin{array}{c}
-1 \text { for } i+j \text { and } i-j \text { even }, i \neq j \\
1 \text { for } i+j \text { and } i-j \text { odd } \\
-\frac{1}{2} \text { for } i=j \neq 0
\end{array} .\right.
$$

Theorem 1. For sake of simplicity, let $\alpha=0$ in model (7) and denote $X^{\prime} \equiv\left(y_{1}^{(m)}, \ldots, y_{T-1}^{(m)}\right)$. If $\sum_{i=0}^{m} \xi_{i, T}^{2}<\frac{1}{2(m+1)}$ then $\frac{1}{T}\left(X^{\prime} X\right) \stackrel{p}{\rightarrow} \sigma^{2} I_{m+1}$, as $T \rightarrow \infty$, where $\sigma^{2}=\lim _{T \rightarrow \infty} \frac{1}{T} \sum_{t=1}^{T} V\left(y_{t-1}\right)$ and $I_{m+1}$ is the identity matrix. 
Proof: Let $\frac{1}{T} \sum_{t=2}^{T} P_{i, T}(t) P_{j, T}(t) y_{t-1}^{2}, i, j=0,1, \ldots, m$ be a generic element of the matrix $\frac{1}{T}\left(X^{\prime} X\right)$. By Bierens (1994), Lemma 9.6.3., page 200, McLeish $(1974,1975)$ and Lemma 1,

$$
\begin{aligned}
& \frac{1}{T} \sum_{t=2}^{T} P_{i, T}(t) P_{j, T}(t) y_{t-1}^{2}=\frac{1}{T} \sum_{t=1}^{T} P_{i, T}(t) P_{j, T}(t) y_{t}^{2}+o_{p}(1) \\
= & (-1)^{i+j} \frac{1}{T} \sum_{t=1}^{T} y_{t-1}^{2}+ \\
& \int_{0}^{1}[(i \pi) \sin (i \pi x) \cos (j \pi x)+(j \pi) \sin (j \pi x) \cos (i \pi x)] \frac{[x T]}{T} \frac{1}{[x T]} \sum_{t=1}^{[x T]} y_{t-1}^{2} d x \\
& +o_{p}(1) \\
& \stackrel{p}{\rightarrow}\left((-1)^{i+j}+a_{i j}\right) \lim _{T \rightarrow \infty} \frac{1}{T} \sum_{t=1}^{T} V\left(y_{t-1}\right), \text { as } T \rightarrow \infty .
\end{aligned}
$$

By Lemma 2,

$$
\frac{1}{T} \sum_{t=2}^{T} P_{i, T}(t) P_{j, T}(t) y_{t-1}^{2} \stackrel{p}{\rightarrow}\left\{\begin{array}{c}
0, i \neq j \\
\frac{1}{2} \lim _{T \rightarrow \infty} \frac{1}{T} \sum_{t=1}^{T} V\left(y_{t-1}\right), i=j \neq 0
\end{array}\right.
$$

as $T \rightarrow \infty$, and the result follows.

In terms of endogeneity, the question is whether the polynomial approximation $m \ll T-1$ generates an error term that is correlated to the regressors. According to Theorem 2, endogeneity is not an issue either in the testing regression (7) for large samples.

Theorem 2. Let $v_{t}=u_{t}+\sum_{i=m+1}^{T-1} \xi_{i} P_{i, T}(t) y_{t-1}$ in model (7) and denote $X^{\prime} \equiv\left(y_{1}^{(m)}, \ldots, y_{T-1}^{(m)}\right)$. If $\sum_{i=0}^{m} \xi_{i, T}^{2}<\frac{1}{2(m+1)}$ then $\frac{1}{T}\left(X^{\prime} v\right) \stackrel{p}{\rightarrow} 0$, as $T \rightarrow \infty$.

Proof: Due to the ortonormality property of the Chebyshev time polynomials, model (4) is, in fact, $y_{t}=\alpha+\xi^{\prime} y_{t-1}^{(m)}+v_{t}$, where $v_{t}=u_{t}+$ $\sum_{i=m+1}^{T-1} \xi_{i} P_{i, T}(t) y_{t-1}$. By the properties of $u_{t}$, endogeneity in model (7), if it exists, is via $P_{i, T}(t) y_{t-1}, i=m+1, \ldots, T-1$. Apart from an $o_{p}(1)$ term, let $\sum_{i=m+1}^{T-1} \xi_{i} \frac{1}{T} \sum_{t=2}^{T} P_{i, T}(t) P_{j, T}(t) y_{t}^{2}, j=0,1, \ldots, m$ denote a generic element of 
the matrix $\frac{1}{T}\left(X^{\prime} v\right)$. By Bierens $(1994)$, McLeish $(1974,1975)$ and Lemma 2 (note that $i \neq j$, in this case),

$$
\sum_{i=m+1}^{T-1} \xi_{i} \frac{1}{T} \sum_{t=2}^{T} P_{i, T}(t) P_{j, T}(t) y_{t}^{2} \stackrel{p}{\rightarrow} 0, \text { as } T \rightarrow \infty,
$$

and the result follows.

In conclusion, we claim that our model (7) is a valid specification for stationary time-series that are well characterized by an $\operatorname{AR}(1)$ model with TV autoregressive coefficient. The least squares estimation procedure has good statistical properties due to the orthonormality property of the Chebyshev time polynomials. Moreover, the corresponding $F_{m, T}$ test statistic is a reliable alternative to the standard tests for parameter constancy. The Chebyshev time polynomials is not the only way to model the TV autoregressive coefficient. For instance, one may use as well the orthogonal Fourier functions suggested by Park and Hahn (1999).

\section{References}

[1] Andrews, D. W. K. (1993), Tests for Parameter Instability and Structural Change with Unknown Change Point. Econometrica 61, 821-856.

[2] Andrews, D. W. K. and Ploberger, W. (1994), Optimal Tests when a Nuisance Parameter is Present Only Under the Alternative. Econometrica 62, 1383-1414.

[3] Bai, J. and Perron, P. (1998), Estimating and Testing Linear Models with Multiple Structural Changes. Econometrica 66, 47-78.

[4] Bierens, H. J. (1994), Topics in Advanced Econometrics: Estimation, Testing and Specification of Cross-Section and Time Series Models. Cambridge University Press.

[5] Bierens and Martins (2009), Time Varying Cointegration. Econometric Theory, forthcoming.

[6] Granger, C. W. J. (2008), Non-Linear Models: Where Do We Go Next - Time Varying Parameter Models?. Studies in Nonlinear Dynamics 6 Econometrics 12, issue 3, Article 1. 
[7] Hansen, B. E. (1992), Tests for Parameter Instability in Regressions with $I(1)$ Processes. Journal of Business and Economic Statistics 10, 321-335.

[8] Hansen, B. E. (2001), The New Econometrics of Structural Change: Dating Breaks in U.S. Labor Productivity. Journal of Economic Perspectives 15, 117-128.

[9] McLeish, D. L. (1974), Dependent Central Limit Theorems and Invariance Principles. Annals of Probability 2, 620-628.

[10] McLeish, D. L. (1975), A Maximal Inequality and Dependent Strong Laws. Annals of Probability 3, 829-839.

[11] Nyblom, J. (1989), Testing for the Constancy of Parameters Over Time. Journal of the American Statistical Association 84, 223-230.

[12] Park, J. Y. and S. B. Hahn (1999), Cointegrating Regressions with Time Varying Coefficients. Econometric Theory 15, 664-703.

[13] Perron, P. (2006), Dealing with Structural Breaks. In Palgrave Handbook of Econometrics 1. Econometric Theory (K. Patterson and T.C. Mills, eds.) 278-352. London:Macmillan.

[14] Quandt, R. (1960), Tests of the Hypothesis that a Linear Regression System Obeys Two Separate Regimes. Journal of the American Statistical Association 55, 324-330. 